\title{
FUNCIONES EJECUTIVAS, POBREZA Y ESTIMULACION COGNITIVA: UN ANDAMIAJE PARA FUTURAS INTERVENCIONES
}

\author{
Executive functions, poverty and cognitive stimulation: \\ a framework for future interventions
}

\author{
María Adela Bertella*; María Paz Grebe*; María Soledad Dalbosco*; \\ Lucía Alba-Ferrara** \\ Universidad Austral
}

\begin{abstract}
Resumen
El presente trabajo presenta el diseño y la implementación de un programa de estimulación de las funciones ejecutivas en una población socialmente vulnerable. La muestra poblacional fue compuesta por 18 niños entre 6 y 11 años edad, de ambos sexos, que asisten a la Posta Sanitaria Las Lilas (Pilar, Argentina), los cuales fueron evaluados antes y después de la implementación del programa con la adaptación argentina de la Escala de Inteligencia para Niños de Wechsler WISC-IV. La estimulación se realizó a través de cuadernillos confeccionados especialmente para trabajar las funciones ejecutivas (memoria de trabajo, flexibilidad cognitiva, inhibición y planificación). Este programa piloto permitió construir un nexo con la comunidad formando un andamiaje institucional, sobre el cual se apoyan nuevos programas de intervención para la misma población.
\end{abstract}

Palabras clave: funciones ejecutivas, estimulación cognitiva, pobreza, vulnerabilidad social.

\begin{abstract}
This paper presents the design and implementation of a executive functions' stimulation program in a socially vulnerable population. The population was composed of 18 children between 6 and 11 years old, of both genders, who attend to Las Lilas Health Post (Pilar, Argentina), which were evaluated before and after the program's implementation with the argentine adaptation of the Wechsler Intelligence Scale for Children WISC-IV. The stimulation was done through a specially made booklet to work the executive functions (working memory, cognitive flexibility, inhibition and planning). This pilot program allowed to build a nexus with the community, forming a institutional framework, on which new intervention programs are supported for the same population.
\end{abstract}

Keywords: Executive functions, cognitive stimulation, poverty, social vulnerability.

\footnotetext{
* Psicóloga, Facultad de Ciencias Biomédicas, Universidad Austral (Buenos Aires, Argentina). mbertella@austral.edu.ar

** Psicóloga. Unidad para el estudio de las Neurociencias y los Sistemas Complejos (ENyS), CONICET (Buenos Aires, Argentina).
} 


\section{INTRODUCCIÓN}

El objetivo del presente trabajo fue desarrollar e implementar un programa piloto de estimulación cognitiva a niños de educación primaria que pertenecen a una población vulnerable. Los objetivos específicos consistieron en estudiar las funciones ejecutivas (comprensión verbal, razonamiento perceptivo, memoria operativa y velocidad de procesamiento) de los niños y diseñar e implementar un programa de estimulación de dichas funciones, evaluando si la intervención mejoraba el desempeño cognitivo y por ende su rendimiento escolar.

Las dos hipótesis de trabajo planteadas consideran que las funciones ejecutivas evaluadas por el WISCIV de los niños que pertenecen a una población vulnerable se encontrarian descendidas en relación a la media poblacional. Asimismo, los niños que participen del programa de intervención presentarian mejoras en el desempeño de sus funciones ejecutivas y consecuentemente en su rendimiento escolar. El contexto en el que se llevó a cabo el programa de estimulación estaba definido por la participación de los niños en talleres de apoyo escolar coordinados por un equipo formado por alumnos universitarios y docentes de la carrera de Psicología de una Universidad privada Argentina .

\section{MARCO TEÓRICO}

\section{Funciones ejecutivas}

Se puede plantear que todo aprendizaje escolar y conducta inteligente requiere de determinadas funciones que hacen posible captar la información que proviene del medio y la regulación de la propia actividad de modo de asegurar la adaptación a las exigencias externas (Rebollo \& Montiel, 2006). Desde las neurociencias, se las conoce como funciones ejecutivas (FE) las cuales requieren, entre otras, de la habilidad para filtrar la información que interfiere con la tarea, involucrarse en conductas dirigidas a un objetivo, anticipar las consecuencias de las propias acciones y el concepto de flexibilidad mental (Denckla, 1996; Luria, 1969; Stuss \& Benson, 1984). Las funciones ejecutivas se describen como un conjunto de habilidades cognitivas vinculadas a la autodirección, la organización de contingencias conductuales a través del tiempo, el uso del lenguaje autodirigido, la formulación de reglas o planes, la gratificación diferida y acciones dirigidas hacia una meta (Ardila \& Ostrosky-Solís, 2008; Denckla, 1996; Stuss \& Benson, 1984). Las mismas refieren no sólo a la solución de problemas, la planeación, la formación de conceptos, el desarrollo e implementación de estrategias y la memoria de trabajo, sino que implican, a su vez, la coordinación de la cognición y de la emoción/motivación. Todas ellas se ponen en juego en la realización de las tareas cotidianas y, por ende, en el aprendizaje escolar. Este tipo de funciones dependen de redes extensas que incluyen diferentes áreas cerebrales, pero es la corteza prefrontal la que juega un papel principal en el control y monitoreo de las conductas (Ardila \& Ostrosky-Solís, 2008).

El concepto funciones ejecutivas (FE) resulta complejo en la búsqueda de una definición exhaustiva para conocer la naturaleza de estos procesos cognitivos con exactitud. A lo largo de los años, se han definido a las FE de diversas maneras según el área de estudio. La neuropsicología se ha referido a ellas desde su localización; la psicología cognitiva como "esa porción de cognición que ocurre después de la percepción, pero antes de la acción" (Arán Filippetti \& López, 2013). No obstante, la principal dificultad para definirlas reside en las diferencias conceptuales que resultan de considerarlas de naturaleza unitaria o diversa. Como lo expresan Verdejo-García y Bechara (2010) uno de los debates más cruciales sobre la naturaleza de las funciones ejecutivas se enmarca en la diversidad funcional de la corteza prefrontal. Desde diferentes perspectivas, las FE podrían considerarse un constructo unitario, o bien pertenecer a un sistema de procesamiento múltiple con distintos componentes independientes aunque interrelacionados. Una mirada unitaria de las FE propone la existencia de un mecanismo subyacente común que permitiría explicar las variaciones en el funcionamiento frontal y así dar cuenta de sus disfunciones (Arán Filippetti \& López, 2013). Por el contrario, desde un marco teórico de procesamientos múltiples, se hace referencia a un constructo integrado por una gran variedad de componentes que se encuentran interrelacionados, pero son diferentes. Es decir, las múltiples capacidades ejecutivas (inhibición, memoria de trabajo, flexibilidad cognitiva, planificación y fluidez verbal y no verbal) actuarían coordinadamente. Estudios recientes 
demuestran la naturaleza diversa pero unitaria de las FE, entendiendo que su estructura está integrada por componentes separados, pero no independientes, lo que supone la existencia de mecanismos subyacentes en común (Collette et al., 2005; Lehto, Juujärvi, Kooistra, \& Pulkkinen, 2003; Miyake et al., 2000).

Desde el marco teórico de este trabajo, se considera esta última conceptualización integradora de la naturaleza de las funciones ejecutivas. Para una mayor comprensión, se utiliza el enfoque impulsado por Goldberg y Brown (Brown, 2006a, 2006b; Goldberg, 2002) de las FE como "director de orquesta" en tanto que son funciones que dirigen la conducta. Lezak (1982) las conceptualizó como aquellas capacidades mentales que se requieren para la formulación de metas, la planificación de cómo alcanzarlas y realizar los planes efectivamente. Actualmente, se consideran a las FE como aquellos procesos cognitivos que permiten realizar conductas complejas a través del establecimiento de metas, siendo esto posible por la interacción de capacidades como la memoria de trabajo, la inhibición cognitiva, la planificación, la flexibilidad cognitiva y la cognición social (Tirapu, García, Ríos, \& Ardila, 2012). Según Lipina y cols., las FE corresponden a funciones cognitivas de alto orden constituidas a su vez por tres componentes integrados: (a) control atencional (atención selectiva y sostenida), (b) flexibilidad cognitiva (memoria de trabajo, cambio atencional, auto monitoreo y transferencia conceptual) y (c) logro de objetivos (iniciación, planificación, resolución de problemas, comportamientos estratégicos). Si se produce una disrupción de alguna de estas habilidades, es posible que los individuos exhiban pobreza atencional o de planificación, dificultades para generar e implementar estrategias, incapacidad para hacer uso de retroalimentación e inflexibilidad en el pensamiento. A su vez, refiere que se han observado correlaciones significativas entre disfunciones ejecutivas y alteraciones del rendimiento aritmético en niños de edad escolar (Lipina, Martelli, Vuelta, Injoque-Ricle y Colombo, 2004).

En esta experiencia piloto, se evaluó a los niños participantes con el WISC IV (Wechsler Intelligence Scale for Children). Dicho instrumento consta de 4 escalas, cada una de las cuales mide diferentes funciones cognitivas. La escala de Comprensión
Verbal busca medir la capacidad para la formación de conceptos, capacidad de razonamiento verbal y el conocimiento adquirido del entorno individual del niño/a. A través de la escala de Memoria Operativa, se evalúa la capacidad para retener temporalmente en la memoria cierta información, trabajar u operar con ella y generar un resultado. Implica atención sostenida, concentración, control mental y razonamiento. Es un componente esencial de otros procesos cognitivos superiores y está relacionada con el rendimiento académico y el aprendizaje. La Velocidad de Procesamiento se refiere a la capacidad para explorar, ordenar o discriminar información visual simple de forma rápida y eficaz. Por último, con la escala de Razonamiento Perceptual se mide el razonamiento fluido, que se manifiesta en tareas que requieren manejar conceptos abstractos, reglas, generalizaciones, relaciones lógicas, en especial sobre material nuevo, el procesamiento espacial y la integración visomotora. Se ha seleccionado este instrumento debido a que posibilita obtener información de funciones ejecutivas como la flexibilidad cognitiva, capacidad de involucrarse en tareas dirigidas a un objetivo, formación de conceptos, planeación y memoria de trabajo.

\section{Pobreza, Vulnerabilidad Social y Educación}

La muestra poblacional incluida en el estudio se caracteriza por encontrarse en una situación de pobreza, y dada dicha condición, se la considera como una población socialmente vulnerable. Sin embargo, cabe destacar que, una población en situación de vulnerabilidad social no necesariamente presenta una condición de pobreza. Para ello, es preciso definir ambas variables. En el Boletín de Pobreza Multidimensional 2010-2015 publicado por el Observatorio de la Deuda Social Argentina de la Universidad Católica Argentina, en base al Enfoque de Derechos (CEPAL, UNICEF), se considera pobreza multidimensional el estar sujeto a privaciones, como la falta de acceso a vivienda, saneamiento, alimentación, salud, información, estimulación y educación. Asimismo, considera que estas privaciones implican una vulneración en el ejercicio de derechos que obstaculizan el desarrollo humano y social de la infancia. En el mismo boletín, se plantea una diferencia respecto de la pobreza moderada (derechos vulnerados) o severa (privación de derechos esenciales) en cuanto a sus consecuencias. En la pobreza moderada, las consecuencias pueden ser 
reversibles en el mediano plazo, mientras que, ante una situación de pobreza severa, sus consecuencias adversas son de difícil reversión en el mediano o corto plazo. Asimismo, la vulnerabilidad social corresponde a aquella situación social de riesgo o dificultad que se manifiestaen contextos socio-históricos y culturalmente determinados inhabilitándole a los grupos afectados la satisfacción de su bienestar, ya sea en su subsistencia como en su calidad de vida. Dicho impedimento puede darse de manera inmediata o en el futuro (Perona \& Rocchi, 2001).

Se clasifican como grupos vulnerables a aquellas personas y grupos en situación de pobreza, de riesgo ambiental (amenazados por desastres naturales), víctimas de violencia, con discapacidad, refugiados y todo grupo que se encuentre en situación de desventaja respecto al resto de los habitantes según la realidad de cada ciudad (Barómetro de la Deuda Social con las Personas Mayores, 2010-2016). Desde esta perspectiva, la muestra poblacional en la que se basa el presente estudio se encuentra en situación de vulnerabilidad, no sólo por su condición de pobreza, sino por su condición de desventaja en cuanto al acceso a la educación y a la salud, a una vivienda digna y al agua potable.

En la literatura científica se encuentran numerosos artículos dedicados a la pobreza y su consecuente impacto negativo en el desarrollo neuro cognitivo, el cual se habría ampliado y profundizado en los últimos años (Musso, 2010). Los hogares en permanente situación de riesgo socio-económico perjudican el aprendizaje de los niños en tanto conlleva que los alumnos no cuenten con los requisitos mínimos exigibles para el aprendizaje de la lectura y escritura, presentando problemas desde el inicio de la etapa escolar (Castro Durán \& Cano González, 2013).

En un estudio realizado por Lipina y cols. (2004) se comparó el desempeño ejecutivo de 247 niños escolarizados de 3 a 5 años de edad provenientes de hogar pobres y no pobres (según Necesidades Básicas Insatisfechas y Satisfechas respectivamente) y de jardines de infantes del Distrito 4 (CABA) y de los distritos escolares de Vicente López y San Isidro
(GBA). Las funciones evaluadas fueron la flexibilidad cognitiva, el control atencional y el logro de objetivos. Los resultados obtenidos indican que el grupo de niños proveniente de hogares pobres obtuvieron un desempeño en las FE menos eficiente en comparación al grupo con necesidades básicas satisfechas (Lipina, Martelli, Vuelta, Injoque-Ricle, \& Colombo, 2004).

Según una investigación inédita realizada por profesionales que trabajan en la Posta Sanitaria Las Lilas (Brunelli, Centeno, Desmery, Sayal, Moll, Morandi, Vera, 2015), el 65\% de la población presenta estudios inferiores a su edad, el $12 \%$ vive en hogares en condiciones de hacinamiento crítico y el $71 \%$ no trabaja. Asimismo, el barrio no cuenta con cloacas ni agua corriente. Los principales accesos están asfaltados, pero las calles son de barro.

\section{METODOLOGÍA}

\section{Muestra Poblacional}

Se partió de una muestra inicial compuesta por 22 niños (9 mujeres y 13 varones) de 6 a 12 años de edad, que concurrieron a la Posta Sanitaria ${ }^{1}$ de manera voluntaria. La muestra final fue de 18 niños de entre 6 y 12 años debido a que 4 niños abandonaron el programa por razones que se desconocen. Los niños asistieron por demanda espontánea e interés al Taller de Apoyo Escolar. Todos los niños participantes y sus respectivos padres firmaron un consentimiento informado para participar voluntariamente en este estudio. El proyecto contó con la aprobación de la Comisión de Bioética de la Universidad Austral, basándose en la Declaración de Helsinki y sus enmiendas.

\section{Instrumento}

Para evaluar las funciones ejecutivas se utilizó la adaptación a la población argentina de la Escala de Inteligencia para Niños de Wechsler WISC-IV (Taborda, Brenlla y Barbenza, 2011). Como fue descripto anteriormente dicho instrumento consta de 4 escalas: Comprensión Verbal (CV), Memoria Operativa (MO), Velocidad de Procesamiento (VP) y Razonamiento Perceptual (RP).

1. Centro de Atención Primaria dependiente de la Facultad de Ciencias Biomédicas de la Universidad Austral ubicado en la localidad de Derqui, Pilar (provincia de Buenos Aires) 
Cada escala está compuesta, a su vez, por subescalas que permiten identificar cuáles son las funciones básicas que se encuentran descendidas y por lo tanto deberán ejercitarse. Se ha seleccionado este instrumento debido a que permite obtener información de funciones ejecutivas como la flexibilidad cognitiva, capacidad de involucrarse en tareas dirigidas a un objetivo, formación de conceptos, planeación y memoria de trabajo. Las mismas están organizadas en cinco índices: CI total, índice de comprensión verbal (ICV), índice de razonamiento perceptual (IRP), índice de memoria operativa (IMO) e índice de velocidad de procesamiento (IVP).

\section{Intervención}

A fin de estimular las $\mathrm{FE}$, se diseñaron $\mathrm{y}$ confeccionaron cuadernillos con ejercicios específicos. Los ejercicios fueron propuestos por los estudiantes de la carrera de Psicología de la Facultad de Ciencias Biomédicas de la Universidad Austral y supervisados por la docente que cumplía la función de coordinadora del Programa de Apoyo Escolar. Los criterios de armado de los cuadernillos se establecieron en función de las escalas del WISC-IV y los niveles se determinaron en función de la edad cronológica de los niños y sus habilidades cognitivas. De este modo, se disponía de sets de cuadernillos para cada una de las dimensiones evaluadas por el WISC IV: CV, MO, VP y RP adaptados a dos niveles. El nivel inicial estaba dirigido a niños de 6 a 9 años y/o que habían obtenido puntuaciones acordes a esta edad cronológica y el nivel medio a niños de 10 a 12 años. Una vez que los niños del nivel inicial lograban completar los cuadernillos sin dificultad, se brindaba luego el cuadernillo de nivel medio. Para más información de los cuadernillos, se sugiere ver "Material Suplementario".

La intervención se llevó a cabo en un total de 12 meses de estimulación. Los intervalos corresponden al receso escolar (vacaciones de verano y de invierno respectivamente). Cada sesión del programa tuvo una duración de dos horas con una frecuencia semanal. Los estudiantes de la carrera de Psicología se desempeñaron como instructores del Programa de Apoyo. Se organizaron equipos de trabajo compuestos por 6 a 10 niños y dos instructores. El número total de estudiantes universitarios que intervinieron como instructores fue de 25 .

\section{Análisis estadístico}

Se compararon los puntajes obtenidos por los niños en la primera evaluación con la media poblacional ajustada por edad mediante un test $t$ de una muestra. Los puntajes crudos que los niños obtuvieron en cada una de las escalas se ajustaron por edad utilizando baremos estadísticos actualizados a fines de determinar el CI de cada niño en cada una de las capacidades. Se realizó un test $\mathrm{t}$ de muestras relacionadas por cada una de las variables evaluadas (ICV, IRP, IMO, IVP) para comparar el desempeño de los niños antes y después de la intervención. Se corrigió el umbral de significación p por comparaciones múltiples.

\section{RESULTADOS Y CONCLUSIONES}

El test $\mathrm{t}$ de una muestra demostró que el grupo de niños incluidos en la muestra poseía un CI total $(\mathrm{M}=76.7, \mathrm{SD}=9.1)$ significativamente más bajo que la media poblacional $\mathrm{t}(21)=-12.1, \mathrm{p}<0.001$

Respecto del test $\mathrm{t}$ de muestras relacionadas para comparar el desempeño de los niños en cada una de las subescalas evaluadas antes y después de la intervención, al realizar correcciones por comparaciones múltiples ajustando el valor $\mathrm{p}$, y considerando los niveles de significación para pruebas de dos colas $(\mathrm{p}<0.001)$, los resultados no arrojaron nivel de significatividad ( $\mathrm{t} \leq$ $3.64, \mathrm{p} \geq .008)$.

Los resultados de la primera evaluación confirman la hipótesis según la cual los niños que pertenecen a una población vulnerable poseen un CI que se encuentra por debajo de la media poblacional.

El análisis estadístico que comparó el desempeño antes y después de la intervención, no demostró diferencias cuantitativamente significativas, pudiéndose mencionar algunas consideraciones relacionadas particularmente con el tamaño de la muestra. Mientras que Aran-Filippetti y colaboradores (2011) evaluaron una muestra de 110 niños, y Ghiglione y colaboradores (2011) una muestra de 98 niños, la muestra del presente estudio contó con los primeros 18 niños que se anotaron en el programa, dado que era una experiencia piloto en la comunidad, lo que pudo haber causado una insuficiencia de poder estadístico para detectar efectos significativos. 
De todos modos el beneficio para los niños que han asistido y asisten al programa ha quedado demostrado en varios aspectos:

La convocatoria del Programa de Apoyo Escolar se fue incrementando desde sus inicios en 2014 cuando comenzaron asistiendo 14 niños, hasta el 2017, en que se encuentran inscriptos 100 niños de los cuales 68 asisten regularmente. La expansión de dicho programa se generó principalmente mediante recomendación de los asistentes y de las maestras de los colegios a los que asistían los niños inscriptos en el programa. Aquellos alumnos que debieron abandonar el programa por incompatibilidad horaria con su horario de escolaridad, sin embargo concurrieron al mismo en la eventual ocasión de suspensión de clases en sus escuelas. El éxito de la convocatoria puede ser interpretado en términos del progreso que los asistentes han realizado a partir de completar los cuadernillos con los que se trabajó para la estimulación de las funciones cognitivas descendidas. Cabe destacar que los padres de los inscriptos manifestaron en forma espontánea a los coordinadores del Programa progresos en sus hijos respecto de la incorporación de una rutina y hábitos de orden para realizar la tarea escolar en la casa. Dichos avances llevaron a los padres a reclamarar una mayor frecuencia del Programa. Los progenitores también notaron avances en la socialización de sus hijos, expresada en la mejora en la resolución de conflictos tanto en los espacios de juego como de trabajo, ejercitando su tolerancia a la frustración, respetando tiempos de espera y mejorado la relación entre pares. Dicho hallazgo coincide con lo percibido y registrado por los estudiantes que se desempeñan como instructores del programa.

Otro aspecto que daría cuenta de los beneficios de la intervención, ha sido la permanencia de los niños participantes en el programa de estimulación y el incremento en la cantidad de niños que se inscriben en el mismo. De la muestra inicial aún continúan asistiendo los 18 niños, habiendo transcurrido más de dos años desde el inicio del mismo. Por el otro lado 80 nuevos niños se sumaron al programa desde la finalización del presente estudio piloto.

Finalmente, y si bien el objetivo principal del Programa ha sido beneficiar a los niños en el desarrollo de sus funciones ejecutivas, no es menos importante destacar que la demanda de los niños y de los padres junto al interés de los estudiantes universitarios y la comunidad educativa en general, han permitido que el mismo tenga un alcance mayor a pesar de las limitaciones metodológicas. El Programa resultó un espacio de contención para los niños y sus padres, quienes tuvieron la oportunidad de consultar por inquietudes en relación al desarrollo general de sus hijos. En varias oportunidades, el programa actuó como espacio de detección temprana de patologías del aprendizaje, orientando e informando a niños y padres sobre la posibilidad de realizar interconsultas en consultorios externos.

En relación con los estudiantes que llevaron a cabo el programa en su implementación, fueron ellos mismos quienes confeccionaron los cuadernillos de estimulación. Además dado que el Programa de Apoyo Escolar se llevó a cabo en la Posta Sanitaria, funcionó como nexo entre la Universidad y la Comunidad de Derqui brindando a los estudiantes de la Carrera de Psicología, la posibilidad de conocer cómo se desempeña un psicólogo en el ámbito comunitario. A su vez, por encontrarse dentro de una Posta Sanitaria, los estudiantes pudieron vivir en campo cómo trabajan los distintos profesionales de la salud de manera interdisciplinaria.

\section{Limitaciones de la intervención}

$\mathrm{Si}$ bien la cantidad de niños que asisten al programa se ha ido incrementando, la muestra inicial con la que se realizó este estudio resultó pequeña, lo cual podría explicar la falta de poder estadístico para obtener resultados cuantitativos significativos. Debido a la repercusión que ha tenido el programa, es esperable que las muestras futuras para próximas investigaciones al menos duplique la muestra actual. Otra posible limitación recae en la frecuencia del programa, considerándose que el efecto de la intervención mejora si es intensiva y continuada en el tiempo. [Arán-Filippetti \& Richaud de Minzi, (2011); Díaz-Granados, Jaramillo de Certain, \& Osorio Villegas, (2010); Ghiglione, Arán Filippetti, Manucci, \& Apaz, (2011); Hermida et. al. (2010); Richaud de Minzi, (2007)]

Por otra parte, deben mencionarse las dificultades de algunos de los participantes al programa para 
mantener regularmente su asistencia, las cuales fueron en parte causadas por causas ambientales (en la zona las lluvias causan anegación de las vías terrestres de acceso).

En síntesis, si bien el objetivo principal del programa ha sido beneficiar a los niños en el desarrollo de sus FE, es importante destacar que el mismo ha tenido otros impactos como el convertirse en un espacio de contención importante tanto para los niños como para sus familias. Este estudio da cuenta de una novel experiencia piloto ajustada al contexto que ha permitido constituir un andamiaje indispensable para intervenciones futuras. Dichas intervenciones apuntan a contemplar en forma integrada las necesidades educativas y de desarrollo psicológico de niños de sectores vulnerables. La inserción de los estudiantes universitarios en dicho trabajo de campo acerca la universidad a la comunidad, haciéndola visible, creando nexos con instituciones y actores como la familia, la comunidad educativa y la sociedad.

\section{REFERENCIAS}

Arán-Filippetti, V., \& Richaud de Minzi, M. C. (2011). Efectos de un programa de intervención para aumentar la reflexividad y la planificación en un ámbito escolar de alto riesgo por pobreza. Universitas Psychologica, 10(2).

Arán Filippetti, V., \& López, M. B. (2013). Las funciones ejecutivas en la clínica neuropsicológica infantil. Psicología desde el Caribe, 30(2), 380415.

Ardila, A., \& Ostrosky-Solís, F. (2008). Desarrollo histórico de las funciones ejecutivas. Revista Neuropsicología, Neuropsiquiatría y Neurociencias, 8(1), 1-21.

Asociación Médica Mundial (2008). Declaración de Helsinski.

Brown, T. E. (2006a). Attention deficit disorder: The unfocused mind in children and adults. Yale University Press.

Brown,T.E.(2006b). Executive functions and attention deficit hyperactivity disorder: Implications of two conflicting views. International Journal of
Disability, Development and Education, 53(1), 35-46.

Brunelli, Centeno, Desmery, Sayal, Moll, Morandi \& Vera. (2015). Analisis sociodemográfico de la población que asiste a la posta sanitaria "Las Lilas". Documento inédito. Universidad Austral.

Castro Durán, L. I., \& Cano González, R. (2013). Pobreza y vulnerabilidad: Factores de riesgo en el proceso educativo. Contextos Educativos, 16.

Collette, F., Van der Linden, M., Laureys, S., Delfiore, G., Degueldre, C., Luxen, A., \& Salmon, E. (2005). Exploring the unity and diversity of the neural substrates of executive functioning. Human brain mapping, 25(4), 409-423.

Denckla, M. B. (1996). A theory and model of executive function: A neuropsychological perspective.

Díaz-Granados, F. I., Jaramillo de Certain, L., \& Osorio Villegas, M. (2010). Efectos de un programa de intervención pedagógica en las aptitudes cognitivas de una muestra de estudiantes del nivel de transición de Barranquilla y Ciénaga (Colombia). Psicología desde el Caribe(26).

Ghiglione, M. E., Arán Filippetti, V., Manucci, V., \& Apaz, A. (2011). Programa de intervención, para fortalecer funciones cognitivas y lingüísticas, adaptado al currículo escolar en niños en riesgo por pobreza. Interdisciplinaria, 28(1), 17-36.

Goldberg, E. (2002). The executive brain: Frontal lobes and the civilized mind. Oxford University Press, USA.

Hermida, M. J., Soledad Segretin, M., Lipina Sol Benarós, S. J., \& Colombo, J. A. (2010). Abordajes neurocognitivos en el estudio de la pobreza infantil: consideraciones conceptuales y metodológicas. International Journal of Psychology and Psychological Therapy, 10(2).

Lehto, J. E., Juujärvi, P., Kooistra, L., \& Pulkkinen, L. (2003). Dimensions of executive functioning: Evidence from children. British Journal of Developmental Psychology, 21(1), 59-80.

Lezak, M. D. (1982). The problem of assessing executive functions. International journal of Psychology, 17(1-4), 281-297. 
Lipina, S. J., Martelli, M. I., Vuelta, B. L., InjoqueRicle, I., \& Colombo, J. A. (2004). Pobreza y desempeño ejecutivo en alumnos preescolares de la ciudad de Buenos Aires (República Argentina). Interdisciplinaria, 21(2), 153-193.

Luria, A. (1969). Frontal lobe syndromes in man.

Miyake, A., Friedman, N. P., Emerson, M. J., Witzki, A. H., Howerter, A., \& Wager, T. D. (2000). The unity and diversity of executive functions and their contributions to complex "frontal lobe" tasks: A latent variable analysis. Cognitive psychology, 41(1), 49-100.

Musso, M. (2010). Funciones ejecutivas: un estudio de los efectos de la pobreza sobre el desempeño ejecutivo. Interdisciplinaria, 27(1), 95-110.

Perona, N., \& Rocchi, G. (2001). Vulnerabilidad y exclusión social. Una propuesta metodológica para el estudio de las condiciones de vida de los hogares. Kairos, 8(8).

Rebollo, M. A., \& Montiel, S. (2006). Atención y funciones ejecutivas. Revista de Neurología, 42(2), S3-S7.
Richaud de Minzi, M. (2007). Fortalecimiento de recursos cognitivos, afectivos, sociales y lingüísticos en niñez en riesgo ambiental por pobreza: un programa de intervención. Avances en investigación en Ciencias del Comportamiento en Argentina, 1, 145-176.

Stuss,D.T.,\&Benson,D.F.(1984).Neuropsychological studies of the frontal lobes. Psychological bulletin, 95(1), 3 .

Taborda, A., Barbenza, C., \& Brenlla, M. E. (2011). Adaptación argentina del WISC-IV Wechsler. Escala de Inteligencia de Wechsler para niños cuarta edición (WISC-IV).

Tirapu, J., García, A., Ríos, M., \& Ardila, A. (2012). Neuropsicología de la corteza prefrontal y las funciones ejecutivas. Barcelona: Viguera.

Verdejo-García, A., \& Bechara, A. (2010). Neuropsicología de las funciones ejecutivas. Psicothema.

Fecha de recepción: $\quad 4$ de abril, 2018

Fecha de aceptación: 30 de mayo, 2018 